\title{
Tissue Response of TM-Joint to Arthritis Induction Methods in Rat
}

\author{
Ding-Han Wang ${ }^{1}$, Mu-Chen Yang ${ }^{1}$, Wun-Eng Hsu², Ming-Lun $\mathrm{Hsu}^{1 *}$ \\ ${ }^{1}$ School of Dentistry, National Yang-Ming University, Taipei, Taiwan \\ 2. Department of Stomatology, Veterans General Hospital, Taipei, Taiwan \\ *E-mail: mlhsu@ym.edu.tw
}

\begin{abstract}
Objective: Pathogenesis of rheumatoid arthritis (RA) related temporomandibular joint disorder remains unclear. Some studies investigated the change of condyle after Complete Freund's Adjuvant (CFA) injection which is similar to osteoarthritis. However, there were few studies to demonstrate effect of TMJ in CIA which mimics RA. The aim of this study was to investigate the TM-joint response of CFA and CIA group in rat model.

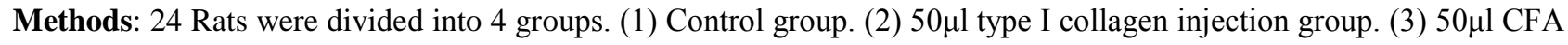
injection group. (4) $25 \mu \mathrm{l}$ type I collagen $+25 \mu \mathrm{l}$ CFA injection group. Drug injection was performed on day 0 and day 14 and sacrificed on day 7, and day 35. After sacrificed, TMJ tissue was collected to do the H\&E stain and investigated the inflammatory (IL-1 $\beta$ and MMP3) gene expression.

Results: TMJ tissue of CFA group demonstrated adaptive bone growth. On the other hand, the subchondral bone of CIA group appeared degenerative process. The trend of IL-1 $\beta$ and MMP3 gene increased in CFA and CIA group compared to control group in synovial tissue and disc $(\mathrm{p}<0.05)$.
\end{abstract}

Conclusion: Within limitation of this study, it's concluded that the CIA method can be used in RA related TMD mechanism research.

Keywords: collagen induced arthritis, rheumatoid arthritis, temporomandibular joint arthritis.

\section{Introduction}

TMD is a disorder commonly encountered in dental clinics. Its symptoms include TMJ and masticatory muscle pain, limitation of mandibular movement and TMJ clicking. It can induce discomfort and pain of the TMJ or masticatory muscles. TMJ arthritis such as rheumatoid arthritis (RA) and osteoarthritis (OA) are also common in clinic1. These diseases induced TMJ arthritis may limit jaw movement, or cause difficulty in chewing, TMJ disc internal derangement and even bone destruction. Because of the patients ${ }^{\text {ee }}$ immune response, bones and joints may be damaged and deformed affecting normal function. Due to inflammation of the joints, the structure of collagen, link proteins and glycosaminoglycan of the articular cartilage cells and extracellular matrix are destroyed. The destruction means TMJ disc cannot maintain its normal shape and function thus failing to assist normal TMJ movement. Clinicians have tried to treat this disorder in different ways, including occlusal adjustment, splint therapy, and prosthetic reconstruction. Recently, injection of extra-cellular matrix such as hyaluronic acid has been proposed as one treatment strategy for management of
TMD. However, pathogenesis of TMD remains controversial and curability. ${ }^{2}$

In previous studies, $\mathrm{OA}$ is the most common arthritis in human body that can also affect TMJ function. ${ }^{3}$ On the other hand, Lin's study in 2007 also postulated that temporomandibular joint abnormalities were found to be highly prevalent $(85.7 \%$ and $74.5 \%$ ) in RA patients by physical and radiologic examination in Taiwan. ${ }^{4} \mathrm{RA}$ is a chronic inflammatory and systemic disorder which may involve joint swelling, joint tenderness, and ultimately bony destruction and joint deformity. ${ }^{5,6}$ In studies of joint disease, Freund's adjuvant (CFA) induced TMJ arthritis has been widely used in physiological, biochemical and histopathological studies7-9. However, pathogenesis of CFA induce TMJ arthritis animal model is more similar to $\mathrm{OA}$ rather than RA. The mechanism of RA related TMJ arthritis pathogenesis remains controversial and curability has not been attained. Therefore, it is necessary to figure out an ideal animal model to mimic RA related TMJ arthritis.

There are few studies to discuss TMJ arthritis animal model with RA, in order to establish TMJ arthritis rat 
model with RA which can mimic human. Method for inducing RA in rat knee for further investigation of the effect of RA for TM-joint was postulated in this study. In Bolon"s study, they try to use different arthritis inducing methods (adjuvant-induced arthritis (AIA), collageninduced arthritis (CIA), and streptococcal cell wallinduced arthritis (SCW)) with different rat strains to evaluate proper method for inducing RA. In the result, Lewis rat is moderate for the disease sensitivity. However,the disease severity in Lewis rats is greatest for AIA. But the immunological change of CIA is similar to RA10. CIA immunize with type II collagen and complete Freund,,s adjuvant (CFA) which can stimulate the antigenpresenting cell (APC) to secrete cytokines to induce the inflammatory response and stimulate the $\mathrm{B}$ cell to produce antibody. ${ }^{8}$ Based on above studies, the aim of this study was trying to development a TMJ-CIA animal model which pathogenesis is similar to RA. This study used the traditional CFA induce arthritis and collagen type I mixed with CFA to mimic RA-like pathway to investigate the TMJ change. These studies can help to clarify (1) the different part of TMJ change during CFA and CIA model. (2) After injection of type I collagen mixed with CFA to induce RA-like pathway whether makes the articular disc become severe degeneration or deformation and displacement.

\section{Methods}

\section{TMJ injection technology}

All the rats followed Institutional Animal Care and Use Committee (IACUC) of National Yang-Ming University and housed under control temperature, on a 12- hour light/dark cycle. In this study, 8 weeks old female Sprague-Dawley rats which were weight 250-320 g were used. $50 \mu \mathrm{l}$ dye (Alcian blue) injection into the superior space of TMJ were used for demonstrate the TMJ injection technology. After injection, rats were sacrificed to check the position.

\section{Drug induced TMJ arthritis model}

24 Rats were divided randomly into 4 groups. Fist group was control group without any experiment. Second group was collagen group with $50 \mu$ l type I collagen injection for clarify whether only type I collagen will lead to rat TMJ arthritis or not. Third group was complete freund's adjuvant (CFA) with $50 \mu \mathrm{l}$ which were emulsion in oil and saline $(1: 1)$. Fourth group was CIA group with $50 \mu \mathrm{l}$ which mixed with type I collagen and CFA (1:1).

Drug injection was performed on day 0 and day 14, and then sacrificed on day 7 and day 35. After sacrificed, day 7 groups TMJ tissue was collected for investigated the inflammatory (IL-1 $\beta$ and MMP3) RNA expression to make sure the TMJ inflammatory response condition. On the other hand, day 7 and day 35 groups were investigated the TMJ tissue change by $H \& E$ stain.

\section{Tissue preparation and histopathology Staining}

One side of rat TMJ tissue was collected for H\&E stain. TMJ tissue of other side was separated to disc, condyle and synovial tissue to make the RNA extraction. RNA was isolated by Trizol (Invitrogen, Carlsbad, CA, USA), purified with Quick-RNATM MiniPrep Kit ZYMO RESEARCH, Irvine, CA, USA). The RNA purity was determined by spectrophotometry, measuring the absorbance of an aliquot at $260 \mathrm{~nm}$ and $280 \mathrm{~nm}$. Total mRNA was reverse transcribed to cDNA using a HighCapacity cDNA Archive kit (Applied Biosystems, Foster City, CA).

\section{Semi-Quantitative PCR}

To investigate the inflammatory (IL-1 $\beta$ and MMP3) gene expression in disc, condyle and synovial tissue by conventional PCR. $\beta$-actin as internal control. The PCR mixture $(20 \mu \mathrm{l})$ contained $150 \mathrm{nM}$ of each primer, $10 \mu \mathrm{l} 2$ $\times$ PCR buffer (Ampliqon III, Odense, Denmark) and $1 \mu \mathrm{l}$ purified DNA. Denaturing $\left(95^{\circ} \mathrm{C}\right)$, annealing $\left(60^{\circ} \mathrm{C}\right)$ and extension $\left(72^{\circ} \mathrm{C}\right)$ times were $1 \mathrm{~min}$ each. The specific product was separated on a $2 \%$ agarose gel and detected after staining with ethidium bromide under UV illumination (Table 1). The procedure for ratio image generation using the ImageJ software

Table 1. Primer used in this study

\begin{tabular}{cccc}
\hline Gene & \multicolumn{1}{c}{$\begin{array}{c}\text { Primer } \\
\text { Sequences }\end{array}$} & $\begin{array}{c}\text { Product } \\
\text { size } \\
(\text { bp })\end{array}$ & Cycles \\
\hline$I L-1 \beta$ & Foward:5'-CAAAAATGCCTCGTGCTGTCT-3' & 130 & 32
\end{tabular}

Reverse:5'-CAGGGATTTTGTCGTTGCTTG-3'

MMP3 Forward:5'-TCCCTGAAACCGTCCAGAAG-3' $173 \quad 32$

Reverse:5'- ATCCACCTTTGTGCCAATGC -3'

$\beta$ - $\quad$ Forward:5'CTATGAGGGTTACGCGCTCC-3' $141 \quad 28$

actin

Reverse:5'- ATGTCACGCACGATTTCCCT-3'

\section{Statistical analysis}

Statistical analysis was performed with SPSS. All data are presented as the mean \pm SEM. Using one-way ANOVA and post-hoc test (Dunnett's) to analyze the data. $\mathrm{P}$ values less than 0.05 were considered as significant.

\section{Results}

SD rats were injected the blue dye into TMJ upper space to demonstrate the injected technology first. The blue color TMJ disc could be observed after removed the surrounding connective tissue of TMJ. These results confirmed the TMJ injection technology could deliver the vehicle into TMJ upper space accurately (Fig.1A). The histology of rat TMJ was investigated by HE stain. Sagittal view of TMJ can divide into three parts: glenoid fossa, TMJ disc and TMJ condyle (Fig.1B). If enlarge the magnification microscope, inside the TMJ disk exist part of chondrocyte cells (Fig. 1C black arrow). The complete structure of TMJ condyle subchondral bone which can be divided into four zones (articular zone, proliferative zone, transition zone and hypertrophic zone) in control group (Fig.1D). 
To develop a TMJ-CIA rat model which mechanism is similar to rheumatoid arthritis, collagen type I injection group was observed for examine that only type I collagen injection will cause TMJ immune response or not first and then compare with CFA and CIA groups. In the 7 days Collagen I group, histology data shown the same condition which compared with control group (Fig.2A). Sagittal view of TMJ disc maintains the regular thickness and part of chondrocyte cells (Fig. 2C black arrow). TMJ condyle subchondral bone also appears four different zones clearly (Fig.2D). However, inflamed cyst could be observed from bright view of 7 days CFA group (Fig.2E). The subchondral bone of 7 days CFA group presented adaptive bone growth (Fig.2F). Thickness of TMJ disc was not change in 7 days CFA group (Fig.2G). If enlarged the view of TMJ condyle subchondral bone, adaptive transition zone bone growth could be investigated (Fig.2H). After inject the type I collagen and CFA mixture, the 7 days CIA group appears more severely inflamed cyst (Fig.2I) and degraded than 7 days CFA group (Fig.2J). Even though TMJ disc of 7 days CIA group maintain the disc thickness, but the chondrocyte cells have disappeared (Fig.2K black arrow). TMJ condyle subchondral bone of 7 days CIA group appear unclearly stratified and severely degraded in hypertrophic zone (Fig.2L).
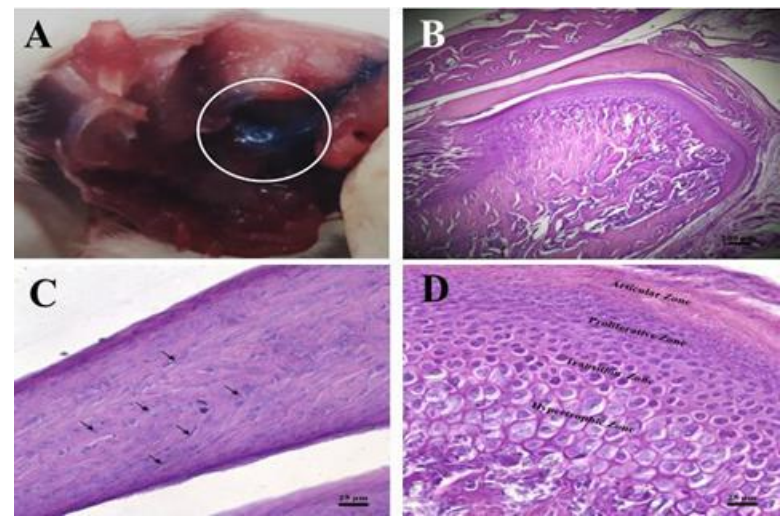

Figure 1. SD rats injection technology checking and histology of control group. (A) The blue color TMJ disc could be observed after removed the surrounding connective tissue of TMJ. (B) Sagittal view of TMJ can divide into three parts: glenoid fossa, TMJ disc and TMJ condyle. Scale bar: $200 \mu \mathrm{m}$. (C) Enlarged view of TMJ disc, fibrocartilage cell can observe clearly (black arrow). Scale bar: $25 \mu \mathrm{m}$. (D) Enlarged view of TMJ condyle subchondral bone can separate to articular zone, proliferative zone, transition zone and hypertrophic zone. Scale bar: $25 \mu \mathrm{m}$.

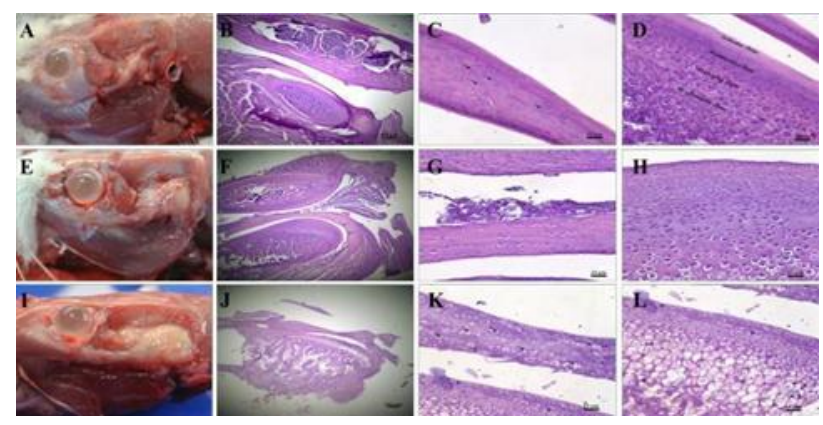

Figure 2. Figure 2. Histology change of 7 days drug induced groups.(A) Bright view of collagen type I group, the TMJ structure could be observed clearly. (B) TMJ sagittal view of collagen type I group. (C) Sagittal view of TMJ disc in collagen type I group which maintains the regular thickness and part of chondrocyte cells (black arrows). (D) Enlarged view of TMJ condyle subchondral bone can separate to 4 layers in collagen type I group. (E) Inflamed cyst could be observed from bright view of CFA group. (F) TMJ sagittal view of CFA group. (G) Sagittal view of TMJ disc in CFA group.

Thickness of TMJ disc was not change. (H) Enlarged view of TMJ condyle subchondral bone in CFA group, adaptive transition zone bone growth could be investigated. (I) Severely inflamed cyst could be observed from bright view of CIA group. (J) TMJ sagittal view of CIA group. (K) Sagittal view of TMJ disc in CIA group. Even though TMJ disc maintain the disc thickness, but the chondrocyte cells has disappeared (black arrows). (L) Enlarged view of TMJ condyle subchondral bone in CIA group, TMJ condyle subchondral bone appears unclearly stratified and severely degraded in hypertrophic zone. Scale bar of figure $2 \mathrm{~B}, 2 \mathrm{~F}, 2 \mathrm{~J}$ are $200 \mu \mathrm{m}$. Scale bar of figure $2 \mathrm{C}, 2 \mathrm{~J}, 2 \mathrm{~K}, 2 \mathrm{D}$, $2 \mathrm{H}, 2 \mathrm{~L}$ are $25 \mu \mathrm{m}$.

In the gene expressions, IL-1 $\beta$ and MMP3 gene increase in CFA and CIA group compared to control group and type I collagen in disc (Fig.3A) and synovial tissue (Fig.3C). There are significant difference in MMP3 of CFA and CIA disc $(F(3,8)=15.715, p=0.034 ; F(3,8)=$ $15.715, \mathrm{p}<0.01)$ and IL-1 $\beta$ of CFA and CIA synovial tissues $(\mathrm{F}(3,8)=9.745, \mathrm{p}=0.012 ; \mathrm{F}(3,8)=9.745, \mathrm{p}=0.02)$. But the expression of these inflammatory genes was lower in condyle (Fig.3B). 


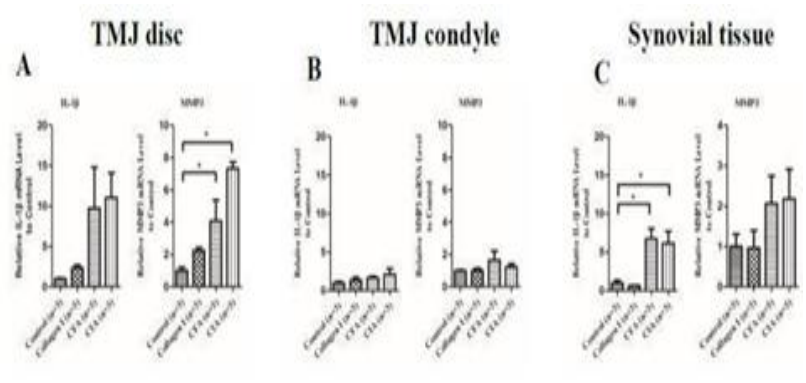

Figure 3. IL-1 $\beta$ and MMP3 gene expression of TMJ tissue after 7 days drug induced. (A) Gene expression of TMJ disc. IL-1ß and MMP3 gene expression of CFA group and CIA group were higher than control group and collagen type I group. (B) TMJ condyle. There is no different between four groups. (C) Gene expression of TMJ Synovial tissue. IL-1 $\beta$ and MMP3 gene expression of CFA group and CIA group were higher than control group and collagen type I group.

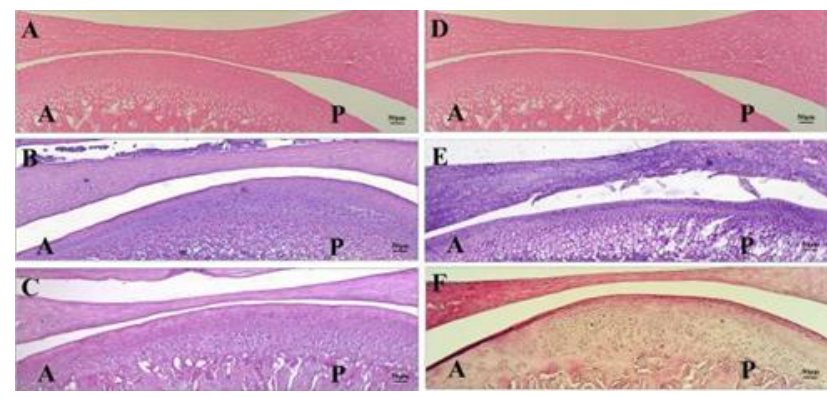

Figure 4. Histology change of 7 days and 35 days drug induced groups. (A, D) TMJ sagittal view of control group.

(B) TMJ sagittal view of 7 days CFA induced group. TMJ condyle appears adaptive bone growth in transition zone.

(C) TMJ sagittal view of 35 days CFA induced group. TMJ condyle transition zone has part of recovery, the TMJ disc has become thinner than control and 7 days CFA group

(E) TMJ sagittal view of 7 days CIA induced group. It appears unclearly stratified and severely degraded in hypertrophic zone.

(F) TMJ sagittal view of 35 days CIA induced group. TMJ disc also become thinner than control and 7 days CIA group. TMJ condyle subchondral bone not only appears unclearly stratified and severely degraded in hypertrophic zone but also shown the irregular cell distribution and abnormal calcification of subchondral bone. *A: anterior. P: posterior.

In addition, 35 days CFA and 35 days CIA groups were also investigated in this study. Compared with control group (Fig.4A), 7 days CFA group appears adaptive transition zone bone growth in TMJ condyle (Fig.4B). However, the TMJ condyle transition zone has part of recovery after 35 days CFA induced. But the TMJ disc has become thinner than control and 7 days' group (Fig.4C). On the other hand, compared with control group (Fig.4D), 7 days CFA group appears unclearly stratified and severely degraded in hypertrophic zone (Fig. 4E). Moreover, The TMJ disc of 35 days CIA group also become thinner than control and 7 days' group. TMJ condyle subchondral bone of 35 days CIA group not only appears unclearly stratified and severely degraded in hypertrophic zone but also shown the irregular cell distribution and abnormal calcification of subchondral bone (Fig. 4F).

These results suggest very different histology change between CFA and CIA group. These results may because of different pathogenesis and lead to different TMJ condyle subchondral bone response.

\section{Discussion}

The aim of this study was try to develop a RA-like TMJ arthritis rat model. CFA and CIA animal models were induced to simulate OA-like and RA-like TMJ arthritis. After drug injection, different time point animal model was investigated the change of TMJ disc, synovial tissue and condyle. In the results, type I collagen injection only group confirm the negative inflammatory response by $\mathrm{HE}$ stains and gene expression of IL-1 $\beta$ and MMP3. In previous study, they usually mixed TMJ tissue together to do the analysis. But if we want to know what happen during TMJ inflammation, it's needed to realize the process of inflammation then maybe can do the treatment before the joint get more serious destruction. In this study, our lab analyzed three part of TMJ tissue which were no any studies have done this before. These results may provide some information to clarify the inflammation response more accurately.

However, use type I collagen and CFA mixture as CIA group inject agent to induce TMJ arthritis appeared different morphology change which compare with other groups. The condyle layer of CIA group became thinner than other groups at day 7 and appeared unclearly stratified and severely degraded in hypertrophic zone but also shown the irregular cell distribution and abnormal calcification of subchondral bone at day 35. On the other hand, 7 days and 35 days CFA induced groups appeared subchondral bone adaptive bone growth of TMJ condyle. In this study, we investigated that the subchondral bone of condyle structure will change first than the disc is also affected during inflammation. These results may explain why TMJ disc still can be observed in severe degeneration or deformation of TMD.

However, in 2001 study have mentioned that the fibrocartilage of condyle changed after CFA injection for 2 days $^{11}$. In addition to in 2012 study used CFA to induced arthritis for 35 days, the fibrocartilage of condyle have still maintained its shape and thickness ${ }^{7}$. These results show the similar appearance with our CFA group and the different appearance in CIA group. Therefore, these results estimate that pathogenesis of CFA and CIA are difference. Besides the different histology change of 
CFA and CIA group in present study, in 1996 study also discovered reduced space and osteophytes were more common in $\mathrm{OA}$, but erosions were more frequently found in RA by X-ray. ${ }^{12}$ Some studies have mentioned that OA has been defined as a "low -inflammatory arthritic condition" and RA has been defined as a "highinflammatory arthritic condition". ${ }^{13}$ Although there can be found a lot of different feasters between OA and RA, ${ }^{14}$ it's still need to find out the most specific marker to identify the difference than can help us to demonstrate the model building.

\section{Conclusions}

Within limitation of this study, it's concluded that the CIA method can be used in RA related TMD mechanism research.

\section{References}

1. Akerman S, Kopp S, Nilner M, Petersson A, Rohlin M. Relationship between clinical and radiologic findings of the temporomandibular joint in rheumatoid arthritis. Oral surgery, oral medicine, and oral pathology. 1988;66(6):63943.

2. Kopp S, Akerman S, Nilner M. Short-term effects of intraarticular sodium hyaluronate, glucocorticoid, and saline injections on rheumatoid arthritis of the temporomandibular joint. Journal of craniomandibular disorders : facial \& oral pain. 1991;5(4):231-8.

3. Ferrazzo KL, Osorio LB, Ferrazzo VA. CT Images of a Severe TMJ Osteoarthritis and Differential Diagnosis with Other Joint Disorders. Case reports in dentistry. 2013:2013:242685.

4. Lin YC, Hsu ML, Yang JS, Liang TH, Chou SL, Lin HY. Temporomandibular joint disorders in patients with rheumatoid arthritis. Journal of the Chinese Medical Association : JCMA. 2007;70(12):527-34.
5. Wong SH, Lord JM. Factors underlying chronic inflammation in rheumatoid arthritis. Archivum immunologiae et therapiae experimentalis. 2004;52(6):37988 .

6. Danoff JR, Moss G, Liabaud B, Geller JA. Total knee arthroplasty considerations in rheumatoid arthritis. Autoimmune diseases. 2013;2013:185340.

7. Wang XD, Kou XX, Mao JJ, Gan YH, Zhou YH. Sustained inflammation induces degeneration of the temporomandibular joint. Journal of dental research. 2012;91(5):499-505

8. Shakya AK, Nandakumar KS. Applications of polymeric adjuvants in studying autoimmune responses and vaccination against infectious diseases. Journal of the Royal Society, Interface / the Royal Society. 2013;10(79):20120536.

9. Holwegner C, Reinhardt AL, Schmid MJ, Marx DB Reinhardt RA. Impact of local steroid or statin treatment of experimental temporomandibular joint arthritis on bone growth in young rats. American journal of orthodontics and dentofacial orthopedics : official publication of the American Association of Orthodontists, its constituent societies, and the American Board of Orthodontics. 2015;147(1):80-8.

10. Bolon B, Stolina M, King C, Middleton S, Gasser J, Zack $\mathrm{D}$, et al. Rodent preclinical models for developing novel antiarthritic molecules: comparative biology and preferred methods for evaluating efficacy. Journal of biomedicine \& biotechnology. 2011;2011:569068.

11. Harper RP, Kerins CA, McIntosh JE, Spears R, Bellinger LL. Modulation of the inflammatory response in the rat TMJ with increasing doses of complete Freund's adjuvant.

12. Gynther GW, Tronje G, Holmlund AB. Radiographic changes in the temporomandibular joint in patients with generalized osteoarthritis and rheumatoid arthritis. Oral surgery, oral medicine, oral pathology, oral radiology, and endodontics. 1996;81(5):613-8.

13. De Souza RF, Lovato da Silva CH, Nasser M, Fedorowicz Z, Al-Muharraqi MA. Cochrane Database Syst Rev; 2012.

14. Sakkas LI, Platsoucas CD. The role of T cells in the pathogenesis of osteoarthritis. Arthritis and rheumatism. 2007;56(2):409-2 